\title{
TERAPIA BIOLÓGICA NA DOENÇA DE CROHN: UM ENFOQUE POSITIVO NOS RESULTADOS TERAPÊUTICOS OBSERVADOS COM OS FÁRMACOS INFLIXIMABE E ADALIMUMABE
}

\author{
BIOLOGICAL THERAPY IN CROHN'S DISEASE: A POSITIVE FOCUS \\ ON THE THERAPEUTIC RESULTS OBSERVED WITH THE \\ ADMINISTRATION OF THE PHARMACEUTICALS INFLIXIMAB AND \\ $A D A L I M U M A B$
}

Jéssica Samara Azevedo Guerra ${ }^{1}$ José Wallysson Soares Pinheiro ${ }^{2}$ Maria Juliana Ferreira dos Santos ${ }^{3}$ Aracele Gonçalves Vieira ${ }^{4}$

RESUMO: Objetivo: Realizar uma revisão integrativa da literatura a respeito da terapia biológica na doença de Crohn, trazendo um enfoque positivo sobre os resultados terapêuticos observados com os fármacos infliximabe e adalimumabe. Método: O presente estudo trata-se de uma revisão integrativa da literatura, com realização entre os meses de junho e dezembro de 2018, tendo a pergunta norteadora: "Como a terapia biológica na doença de Crohn promove resultados terapêuticos positivos com a utilização dos fármacos infliximabe e adalimumabe?". O levantamento bibliográfico foi feito por meio do recurso digital Internet, nas bases de dados SciELO (Scientific Electronic Library Online) e LILACS (Literatura LatinoAmericana e do Caribe em Ciências da Saúde). Para a busca dos artigos científicos, utilizaram-se mecanismos de cruzamento com os seguintes descritores: "Doença de Crohn", "Infliximabe", "Adalimumabe" e "Terapia Biológica". Dentre os critérios de inclusão, estavam: artigos publicados nos idiomas português, inglês e espanhol; que disponibilizassem, de modo gratuito, seu conteúdo de forma íntegra; fossem publicados e indexados de 2009 a 2018; e atendessem ao que se propunha o objeto de investigação deste estudo, excluindo-se formatos documentais de trabalho de conclusão de curso, dissertação e tese. Resultados: A doença de Crohn engloba o grupo das doenças inflamatórias intestinais. Por não ter patogênese ainda bem

\footnotetext{
${ }_{1}^{1}$ Pós-graduanda pela Faculdade Santa Maria (FSM) - Cajazeiras (PB).

${ }^{2}$ Pós-graduanda pela Faculdade Santa Maria (FSM) - Cajazeiras (PB),

${ }^{3}$ Acadêmica do curso de Graduação em Fisioterapia da Faculdade Santa Maria (FSM), Cajazeiras (PB). Email: pqjubs@outlook.com.

Fisioterapeuta. Especialista em Docência do Ensino Superior (FSM) e em Saúde da Família (FACISA). Mestre em Saúde e Sociedade (UERN). Docente da Faculdade Santa Maria (FSM). Email: aracagv@hotmail.com.
} 
esclarecida, seu tratamento é tido como uma questão desafiadora. A terapia biológica, através dos fármacos infliximabe e adalimumabe, tem promovido resultados terapêuticos positivos para esta condição patológica. Sendo observado melhoras, como a diminuição dos surtos de exacerbação e suas frequências, controle da doença, indução da remissão e redução de hospitalizações, muitas vezes associados a processos orgânicos como indução da apoptose de linfócitos $T$, recuperação da integridade da barreira epitelial e indução da mobilidade de fibroblastos presentes no intestino, culminando na cicatrização de lesões. Conclusão: A doença de Crohn, além de promover mudanças potencialmente negativas na qualidade de vida dos seus portadores, ainda se constitui como um desafio para o estabelecimento preciso de suas bases fisiopatológicas, diagnósticas e terapêuticas. Nesta perspectiva, investigar fármacos utilizados no seu tratamento, como infliximabe e adalimumabe, é de grande relevância, pois, mediante tais conhecimentos, tanto o profissional farmacêutico, através dos serviços de farmácia clínica e atenção farmacêutica, quanto à equipe multidisciplinar de saúde, podem contribuir com a obtenção de melhores desfechos clínicos.

Palavras chave: Doença de Crohn. Infliximabe. Adalimumabe. Terapia Biológica.

ABSTRACT: Objective: To carry out an integrative review of the literature on biological therapy in Crohn's disease, bringing a positive focus on the therapeutic results observed with the drugs infliximab and adalimumab. Method: The present study is an integrative review of the literature, carried out between June and December 2018, with the guiding question: "How does biological therapy in Crohn's disease promote positive therapeutic results with the use of drugs infliximab and adalimumab?" The bibliographic survey was done using the Internet digital resource, in the SciELO (Scientific Electronic Library Online) and LILACS (Latin American and Caribbean Literature in Health Sciences) databases. For the search of the scientific articles, crossing mechanisms were used with the following descriptors: "Crohn's disease", "Infliximab", "Adalimumab" and "Biological Therapy". Among the inclusion criteria were: articles published in Portuguese, English and Spanish; to make their contents available in a free way; published and indexed from 2009 to 2018; and to attend to what was proposed the research object of this study, excluding documentary work formats of course completion, dissertation and thesis. Results: Crohn's disease encompasses the group of inflammatory bowel diseases. Because it does not have pathogenesis still well understood, its treatment is considered a challenging question. Biological therapy, through the drugs infliximab and adalimumab, has promoted positive therapeutic results for this pathological condition. Improvements were observed, such as the reduction of exacerbation outbreaks and their frequencies, disease control, induction of remission and reduction of hospitalizations, often associated with organic processes such as induction of $T$ lymphocyte apoptosis, recovery of the integrity of the epithelial barrier and induction of mobility of fibroblasts present in the intestine, culminating in the healing of lesions. Conclusion: Crohn's disease, besides promoting potentially negative changes in the quality of life of its patients, still constitutes a challenge for the precise establishment of its pathophysiological, diagnostic and therapeutic bases. In this perspective, investigating drugs used in their treatment, such as infliximab and adalimumab, is of 
great relevance because, through such knowledge, both the pharmaceutical professional, through the services of clinical pharmacy and pharmaceutical care, and the multidisciplinary health team, can contribute with better clinical outcomes.

Keywords: Crohn'sdisease. Infliximab. Adalimumab. Biological Therapy. 


\section{INTRODUÇÃO}

A doença de Crohn (DC) caracteriza-se como uma doença inflamatória intestinal (DII). Com progressão crônica e exibição de inflamação granulomatosa transmural, tal condição patológica pode afetar qualquer parte do trato gastrintestinal (TGI), embora envolva mais comumente a porção terminal do íleo. Ademais, juntamente com todos estes achados, é também observada a presença de ulcerações descontínuas, frequentemente associadas a estenoses e fístulas (LAW; LI, 2014; SCOLARO et al., 2014).

A patogênese da DC ainda não é bem esclarecida. Assim, não há uma etiologia definida para compreendê-la. No entanto, sabe-se que fatores condicionantes permissivos aos genes, meio ambiente, flora entérica, agentes infecciosos, além de um processo inflamatório imunomediado de forma exacerbada, podem estar envolvidos em sua etiopatogenia (VICTORIA; SASSAK; NUNES, 2009).

Ao longo de seu curso, a DC é marcada por períodos de atividade e remissão, com sintomas que atingem tanto o TGI quanto outras regiões do corpo (HARDT et al., 2012; LARANJEIRA et al., 2015).

Sua apresentação clínica é complexa, com manifestações que às vezes são subclínicas, podendo evoluir para casos graves e acometer locais extensos ou múltiplos, sob a forma de perfuração livre, massas intra-abdominais ou fístulas (NOGUEIRA et al., 2013). Entre os sintomas clínicos mais observados, estão: diarreia, dor abdominal, perda de peso, febre, mucorreia, hematoquezia, sangramento gastrintestinal e fadiga (BECHARA et al., 2015; BACK et al., 2017).

No que concerne aos aspectos farmacoterapêuticos, a terapia biológica (TB) tem se mostrado altamente eficaz nos estágios moderado e grave da DC, principalmente no desenvolvimento da indução da resposta remissiva e sua manutenção. Dentre os principais agentes disponíveis desta classe farmacológica, figuram-se os seguintes fármacos: infliximabe (IFX) e adalimumabe (ADA) (SALESCAMPOS et al., 2015). 
Esta alternativa terapêutica consiste no emprego de substâncias com atividades farmacológicas pautadas na modificação da resposta biológica do intestino, de modo a promover o bloqueio de citocinas específicas que modulam o processo inflamatório na existência de uma condição conhecida como pleomorfismo genético, cujo o gene mais afetado é o NOD2/CARD15, localizado no cromossomo 16 (SANTOS JÚNIOR, 2011).

O IFX é um anticorpo monoclonal, de aspecto quimérico e relacionado à classe da imunoglobulina G - subclasse 1 (lgG1). Apresentando uma composição molecular distinta (75\% humana e $25 \%$ murina), tal fármaco inibe o fator de necrose tumoral alfa (TNF- $\alpha$ ), o que o faz ser classificado como agente anti-TNF- $\alpha$, e demonstra efeitos benéficos na cicatrização de fístulas na DC (DENADAl; TEIXEIRA; SAAD-HOSSNE, 2012; SALES-CAMPOS et al., 2015).

Com relação ao ADA, este tem sua composição molecular de origem totalmente humana, sendo bastante utilizado em pacientes não responsivos ao IFX, principalmente os que são intolerantes às sequências murinas presentes em sua estrutura. É notório também destacar que portadores de DC, apresentando responsividade ao tratamento com o fármaco em questão, podem alcançar a fase de remissão clínica profunda, que se configura através de desfechos favoráveis e melhor qualidade de vida (SALES-CAMPOS et al., 2015).

Tendo em vista o que foi acima relatado, o interesse em trazer esta temática pautou-se na investigação da positividade dos resultados terapêuticos promovidos pela TB nos indivíduos acometidos com DC, sobretudo os que apresentam a doença em estágios que variam de moderado a grave, reafirmando assim a importância de tais fármacos (IFX e ADA).

Assim, mediante o exposto, é de suma importância para avaliar a amplitude do espectro terapêutico promovido pela TB, atentando-se para a sua aplicabilidade clínica e demais aspectos inerentes a tal, como grau de atividade da doença, responsividade a outros agentes farmacológicos, tempo de tratamento, entre outros.

Desta forma, o presente estudo tem como objetivo principal realizar uma revisão integrativa da literatura a respeito da terapia biológica na doença de Crohn, trazendo um enfoque positivo sobre os resultados terapêuticos observados com os fármacos infliximabe e adalimumabe. 


\section{MÉTODO}

O presente estudo configura-se como uma revisão integrativa da literatura. Que, para nortear os aspectos investigativos deste estudo, a inquietação partiu da seguinte pergunta: Como a terapia biológica na doença de Crohn promove resultados terapêuticos positivos com a utilização dos fármacos infliximabe e adalimumabe?

O levantamento bibliográfico foi realizado por meio do recurso digital Internet, onde, mediante rastreio nas bases de dados SciELO (Scientific Electronic Library Online) e LILACS (Literatura Latino-Americana e do Caribe em Ciências da Saúde), obteve-se material necessário para subsidiar e discutir a temática. Ademais, no que concerne à busca dos artigos científicos, utilizou-se mecanismos de cruzamento (doença de Crohn + operador booleano and + infliximabe, adalimumabe ou terapia biológica) com os seguintes descritores e seus equivalentes na língua inglesa: "Doença de Crohn", "Infliximabe", "Adalimumabe" e "Terapia Biológica". A nível temporal, a coleta do material ocorreu entre os meses de junho e dezembro de 2018.

Como critérios de inclusão para a seleção do acervo bibliográfico, foram utilizados: artigos publicados nos idiomas português, inglês e espanhol; que disponibilizassem, de modo gratuito, seu conteúdo de forma íntegra; fossem publicados e indexados nos referidos bancos de dados nos últimos dez anos; e atendessem ao que se propunha o objeto de investigação deste estudo. Para os critérios de exclusão, lançou-se mão do seguinte crivo: formatos documentais de trabalho de conclusão de curso, dissertação e tese.

Feito isso, obteve-se os seguintes resultados: 
Figura 01: Resultados dos artigos encontrados nas bases de dados SciELO e LILACS.

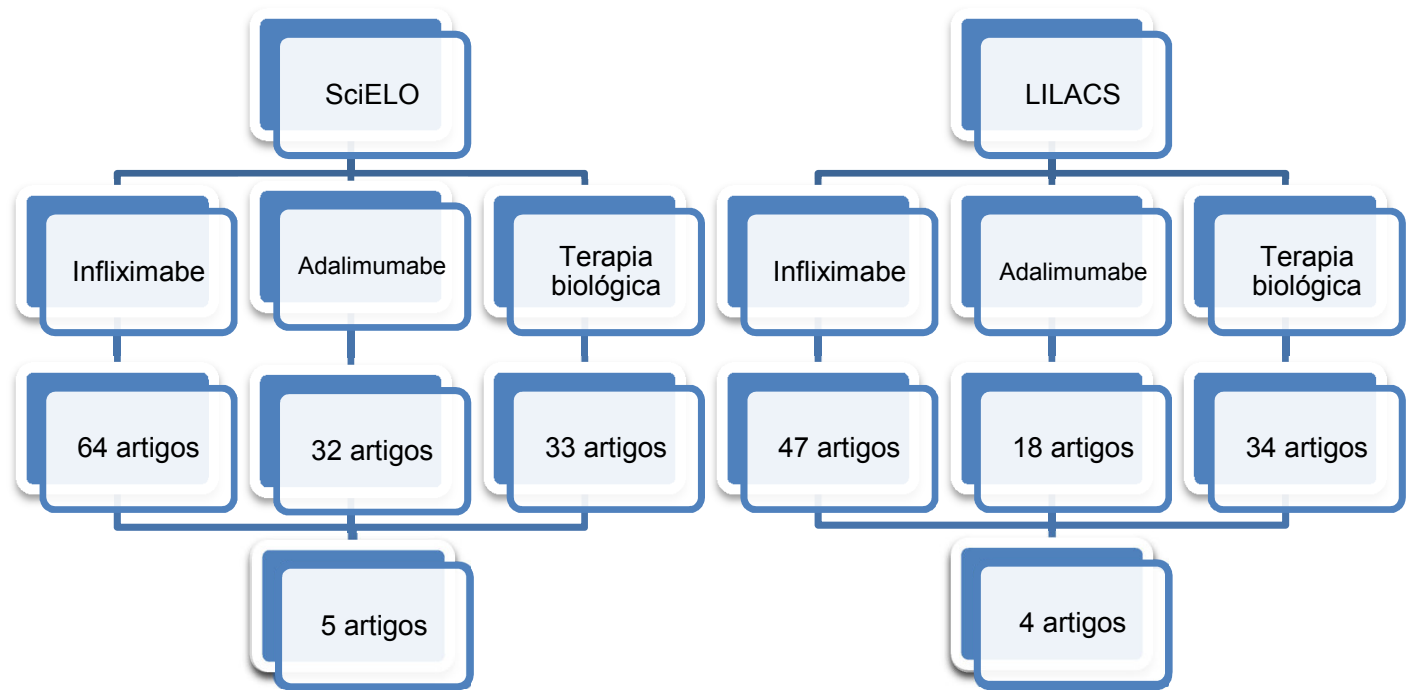

Fonte: Dados da pesquisa (2018)

Realizadas estas etapas, por meio de busca exaustiva, partiu-se para a elaboração do conteúdo, analisando e discutindo o que foi coletado, a fim de contribuir com o assunto investigado.

\section{RESULTADOS}

Neste estudo, configurado como uma revisão integrativa da literatura foram colocados sob análise, para subsidiar as seções (Resultados e Discussão), nove artigos científicos, os quais atenderam a todos os critérios de inclusão previamente estabelecidos.

No Quadro 01, com disposição obedecendo à divisão em quatro colunas, estão elencados os principais marcadores metodológicos dos registros literários componentes da amostra, a saber: título; base de dados/periódico e ano de publicação. No Quadro 02 está elencado, respectivamente, o(s) autor (es); objetivos e principais resultados encontrados. 
Quadro 01: Apresentação da síntese de artigos incluídos na revisão. Título/Base de dados/Ano.

\begin{tabular}{|c|c|c|c|}
\hline $\mathbf{N}^{\circ}$ & TÍTULO & BASE & ANO \\
\hline 1 & $\begin{array}{l}\text { Uso do infliximabe na consulta de } \\
\text { gastrenterologia pediátrica. }\end{array}$ & $\begin{array}{l}\text { SciELO/Jornal Português } \\
\text { de Gastrenterologia. }\end{array}$ & 2012 \\
\hline 2 & $\begin{array}{l}\text { Adalimumabe na terapia de manutenção } \\
\text { por um ano na doença de Crohn: } \\
\text { resultados de um estudo observacional } \\
\text { unicêntrico latino-americano. }\end{array}$ & $\begin{array}{l}\text { LILACS/Arquivos } \\
\text { Gastrenterologia. }\end{array}$ & 2014 \\
\hline 3 & $\begin{array}{l}\text { Remissão perianal completa com terapia } \\
\text { combinada (sedenhos e agentes } \\
\text { biológicos) na doença de Crohn: um estudo } \\
\text { brasileiro observacional e multicêntrico. }\end{array}$ & $\begin{array}{l}\text { LILACS/Arquivos } \\
\text { Gastrenterologia. }\end{array}$ & 2014 \\
\hline 4 & $\begin{array}{l}\text { Tratamento da doença de Crohn com } \\
\text { infliximabe: primeira opção? }\end{array}$ & $\begin{array}{l}\text { LILACS/ABCD } \\
\text { Brasileiros de Cirurgia } \\
\text { Digestiva. }\end{array}$ & 2009 \\
\hline 5 & $\begin{array}{l}\text { Adalimumabe na indução da remissão da } \\
\text { doença de Crohn: resultados de uma série } \\
\text { de casos multicêntricos. }\end{array}$ & $\begin{array}{l}\text { LILACS/Jornal } \quad \text { de } \\
\text { Coloproctologia. }\end{array}$ & 2011 \\
\hline 6 & $\begin{array}{l}\text { Infliximabe na doença de Crohn: } \\
\text { experiência clínica de um centro terciário } \\
\text { paulista. }\end{array}$ & $\begin{array}{l}\text { SciELO/Revista Brasileira } \\
\text { de Coloproctologia. }\end{array}$ & 2009 \\
\hline 7 & $\begin{array}{l}\text { Eficácia do adalimumabe para o tratamento } \\
\text { de manifestações extraintestinais da } \\
\text { doença de Crohn. }\end{array}$ & $\begin{array}{l}\text { SciELO/Revista } \\
\text { Espanhola de } \\
\text { Enfermidades Digestivas. }\end{array}$ & 2012 \\
\hline 8 & $\begin{array}{l}\text { Efeito dos antagonistas do fator de necrose } \\
\text { tumoral alfa no estresse oxidativo em } \\
\text { pacientes com doença de Crohn. }\end{array}$ & $\begin{array}{l}\text { SciELO/Jornal Mundial } \\
\text { de Gastrenterologia. }\end{array}$ & 2015 \\
\hline 9 & $\begin{array}{l}\text { Experiência do uso de terapia biológica em } \\
\text { pacientes com enfermidade intestinal } \\
\text { inflamatória em um hospital público. }\end{array}$ & $\begin{array}{l}\text { SciELO/Gastrenterologia } \\
\text { Latino-americana. }\end{array}$ & 2015 \\
\hline
\end{tabular}

Quadro 02: Apresentação da síntese de artigos incluídos na revisão integrativa. Autor/Objetivos/Principais Resultados.

\begin{tabular}{|c|c|c|c|}
\hline $\mathbf{N}^{\circ}$ & AUTOR & OBJETIVOS & PRINCIPAIS RESULTADOS \\
\hline 1 & $\begin{array}{l}\text { MARQUES } \\
\text { et al. }\end{array}$ & $\begin{array}{lr}\text { Avaliar a resposta } \\
\text { clínica e os efeitos } \\
\text { adversos } & \text { da } \\
\text { terapêutica } & \text { com } \\
\text { infliximabe } & \text { na } \\
\text { doença } & \\
\text { inflamatória } & \\
\text { intestinal } & \text { em }\end{array}$ & $\begin{array}{l}\text { Dos } 6 \text { doentes que realizam terapêutica } \\
\text { monoclonal com infliximabe, } 5 \text { apresentam } \\
\text { doença de Crohn moderada a grave e um } \\
\text { colite ulcerosa. Verificou-se remissão clínica } \\
\text { em } 5 \text { doentes. Observou-se resolução das } \\
\text { alterações analíticas após } 6 \text { meses de } \\
\text { terapêutica. Verificaram-se } 2 \text { reações } \\
\text { alérgicas ligeiras e uma elevação transitória }\end{array}$ \\
\hline
\end{tabular}




\begin{tabular}{|c|c|c|c|}
\hline & & $\begin{array}{l}\text { Dentes } \\
\text { ediátricos. }\end{array}$ & das transaminases. \\
\hline 2 & $\begin{array}{l}\text { KOTZE } \\
\text { al. }\end{array}$ & $\begin{array}{l}\text { Analisar as taxas } \\
\text { de remissão após } \\
12 \quad \text { meses de } \\
\text { terapia } \\
\text { adalimumabe } \\
\text { portadores } \\
\text { doença de Crohn. }\end{array}$ & $\begin{array}{l}\text { Cinquenta pacientes, com média de idade } \\
\text { de } 35 \text { anos no início da terapia, foram } \\
\text { incluídos. As taxas de remissão após um } \\
\text { ano foram de } 54 \% \text { (análise de imputação de } \\
\text { não-resposta) e } 88 \% \text { (análise de última } \\
\text { observação considerada). A remissão } \\
\text { clínica ocorreu em } 69,23 \% \text { dos pacientes } \\
\text { com infliximabe prévio e 94,59\% nos } \\
\text { virgens de infliximabe }(P=0,033) \text {. }\end{array}$ \\
\hline 3 & $\begin{array}{l}\text { KOTZE } \\
\text { al. }\end{array}$ & $\begin{array}{lr}\text { Analisar as taxas } \\
\text { de } & \text { remissão } \\
\text { perianal completa } \\
\text { com a terapia } \\
\text { combinada r na } \\
\text { doença de } & \text { Crohn } \\
\text { perianal } & \\
\text { fistulizante. } & \end{array}$ & $\begin{array}{l}\text { Foram incluídos } 78 \text { pacientes, } 44(55,8 \%) \\
\text { mulheres, com média de idade de } 33,8 \\
( \pm 15) \text { anos. A maior parte dos pacientes foi } \\
\text { tratada com infliximabe, } 66,2 \% \text {, do que com } \\
\text { adalimumabe, } 33,8 \% \text {. Fístulas complexas } \\
\text { foram observadas } 52 / 78(66,7 \%) \text { pacientes. } \\
\text { Após um seguimento médio de } 48,2 \text { meses, } \\
41 / 78(52,6 \%) \text { pacientes apresentaram } \\
\text { remissão perianal completa (IC } 95 \% \text { : } \\
43,5 \%-63,6 \%) \text {. Recorrência foi observada } \\
\text { em quatro }(9,8 \%) \text { pacientes (IC } 95 \%: 0,7 \%- \\
18,8 \%) \text { em um período médio de } 74,8 \\
\text { meses para sua ocorrência. }\end{array}$ \\
\hline 4 & $\begin{array}{l}\text { MALHEIRO } \\
\text { S et al. }\end{array}$ & $\begin{array}{l}\text { Observar os } \\
\text { resultados do } \\
\text { tratamento da } \\
\text { doença de } \\
\text { com o anti-fator de } \\
\text { necrose tumoral } \\
\text { alfa. }\end{array}$ & $\begin{array}{l}\text { No tratamento inicial, } 76 \% \text { dos pacientes } \\
\text { responderam ao anticorpo. Observou-se } \\
\text { que, após a primeira dose da medicação, os } \\
\text { com mais de } 10 \text { anos de doença e } \\
\text { submetidos à operação abdominal, tiveram } \\
\text { resultado satisfatório, semelhantemente } \\
\text { àqueles doentes com menos de cinco anos } \\
\text { de doença e não operados }(62,5 \% \text { e } 80 \% \text {, } \\
\text { respectivamente, dos doentes que } \\
\text { melhoraram), sendo este resultado } \\
\text { estatisticamente significativo. }\end{array}$ \\
\hline 5 & $\begin{array}{l}\text { KOTZE } \\
\text { al. }\end{array}$ & $\begin{array}{lr}\text { Determinar o papel } \\
\text { do adalimumabe } \\
\text { na indução da } \\
\text { remissão r na } \\
\text { doença de } & \text { Crohn } \\
\text { em uma série } \\
\text { brasileira } \\
\text { casos. }\end{array}$ & $\begin{array}{l}54 \text { pacientes foram analisados }(29 \\
\text { mulheres), com média de idade de } 36,72 \\
\text { (15 a 62) anos. Após a dose de indução da } \\
\text { remissão, } 26 \text { pacientes }(48,14 \%) \\
\text { apresentaram resposta total (remissão } \\
\text { clínica), } 26(48,14 \%) \text { tiveram reposta parcial } \\
\text { e } 2 \text { (3,72\%) foram não-respondedores } \\
\text { primários. Após seguimento médio de } 9,83 \\
\text { (entre } 2 \text { e } 28 \text { meses, } 17 \text { pacientes } \\
\text { (31,48\%) apresentaram efeitos adversos (o } \\
\text { mais comum foi dor no local da injeção em } \\
7 \text { pacientes - 12,96\%). }\end{array}$ \\
\hline 6 & TORRES et & Caracterizar & Houve predomínio de pacientes do sexo \\
\hline
\end{tabular}




\begin{tabular}{|c|c|c|c|}
\hline & al. & $\begin{array}{l}\text { indicações clínicas } \\
\text { do infliximabe na } \\
\text { doença de Crohn } \\
\text { em um serviço } \\
\text { referencial } \\
\text { paulista, avaliando } \\
\text { padrões r de } \\
\text { resposta er a } \\
\text { efetividade r do } \\
\text { tratamento através } \\
\text { do Índice de } \\
\text { Atividade r da } \\
\text { doença de Crohn } \\
\text { (CDAl). }\end{array}$ & $\begin{array}{l}\text { feminino }(67 \%) \text {, com média de idade de } 33 \\
\text { anos. As indicações mais frequentes foram } \\
\text { fístulas perianais (48\%). Resposta clínica } \\
\text { total à droga ocorreu em } 43 \% \text { dos pacientes } \\
\text { e resposta parcial em } 47 \% \text {; a diferença da } \\
\text { média do CDAl entre os grupos antes e } \\
\text { após o tratamento foi de } 244,61 \text { pontos } \\
(p<0,0005) \text {. }\end{array}$ \\
\hline 7 & $\begin{array}{l}\text { BARREIRO } \\
\text {-DE- } \\
\text { ACOSTA; } \\
\text { LORENZO; } \\
\text { DOMÍNGU } \\
\text { EZ- } \\
\text { MUÑOZ. }\end{array}$ & $\begin{array}{l}\text { Avaliar a eficácia } \\
\text { do adalimumabe } \\
\text { (ADA) para } \\
\text { tratamento de } \\
\text { manifestações } \\
\text { extraintestinais em } \\
\text { pacientes com } \\
\text { doença de Crohn. }\end{array}$ & $\begin{array}{l}\text { No sexto mês, } 76,2 \% \text { dos pacientes } \\
\text { apresentaram remissão ou resposta na } \\
\text { doença de Crohn (33,3\% de remissão e } \\
42,9 \% \text { de qualquer resposta). } \\
\text { Manifestações extraintestinais mostraram } \\
\text { um curso paralelo com a doença de Crohn } \\
\text { na maioria dos casos, e mostraram } \\
\text { remissão ou resposta em } 66,7 \% \text { dos } \\
\text { pacientes ( } 38,1 \% \text { de remissão e } 28,5 \% \text { de } \\
\text { qualquer reposta). Os pacientes com } \\
\text { qualquer resposta da sua condição de } \\
\text { manifestação extraintestinal foram mais } \\
\text { jovens do que aqueles sem resposta } \\
\text { (p=0,04). Nenhuma relação foi encontrada } \\
\text { entre sexo, tabaco, história familiar de } \\
\text { doença inflamatória intestinal, fenótipo e } \\
\text { tratamento prévio com anti-fator de necrose } \\
\text { tumoral, e resolução de manifestação } \\
\text { extraintestinal. }\end{array}$ \\
\hline 8 & $\begin{array}{l}\text { YAMAMOT } \\
\text { O; CHIBA; } \\
\text { MATSUMO } \\
\text { TO }\end{array}$ & $\begin{array}{lr}\text { Investigar } & \text { as } \\
\text { mudanças } & \text { no } \\
\text { estresse oxidativo } & \text { na doença de } \\
\text { Crohn (DC) } & \text { antes } \\
\text { e depois do } & \text { dratamento rom } \\
\text { tranti-fator r de } & \text { necrose tumoral } \\
\text { alfa (TNF- } \alpha \text { ). }\end{array}$ & 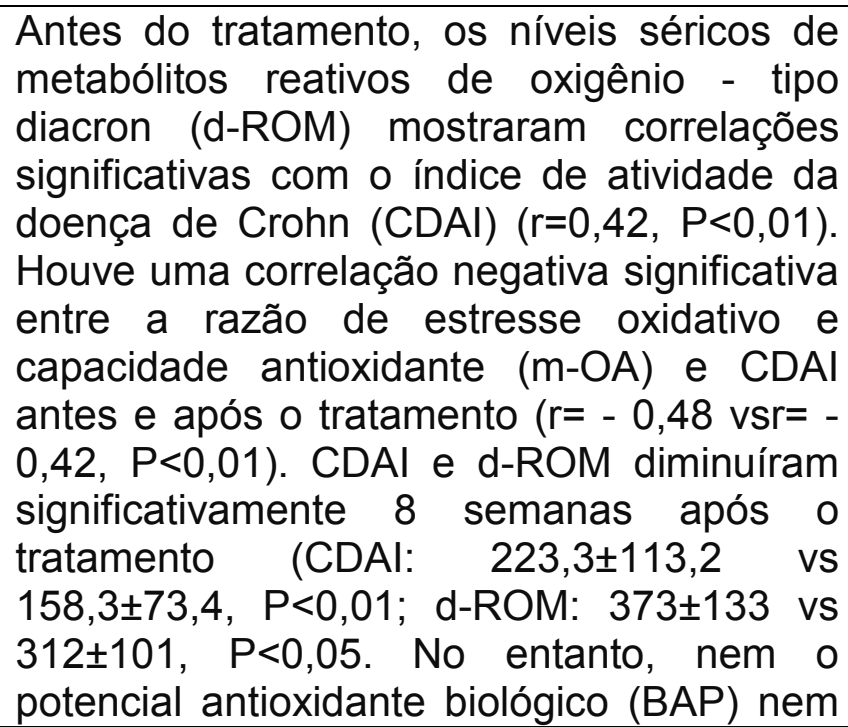 \\
\hline
\end{tabular}




\begin{tabular}{|c|c|c|c|}
\hline & & & $\begin{array}{l}\text { o OA mudaram significativamente. Em } \\
\text { pacientes que haviam respondido ao } \\
\text { tratamento em } 8 \text { semanas, os níveis de d- } \\
\text { ROM, BAP e m-OA antes do tratamento não } \\
\text { diferiram significativamente entre os } \\
\text { pacientes com e sem resposta. }\end{array}$ \\
\hline 9 & $\begin{array}{l}\text { ROJAS T. } \\
\text { et al. }\end{array}$ & $\begin{array}{lr}\text { Relatar } & \text { a } \\
\text { experiência } & \text { com o } \\
\text { uso } & \text { de } \\
\text { infliximabe/adalimu } \\
\text { mabe r em } \\
\text { pacientes r com } \\
\text { doenças } \\
\text { inflamatórias } \\
\text { intestinais (IBD) } \\
\text { em um hospital } \\
\text { público. }\end{array}$ & $\begin{array}{l}\text { Quinze dos } 162 \text { pacientes, com idade entre } \\
17 \text { e } 52 \text { anos de idade ( } 7 \text { mulheres), foram } \\
\text { incluídos. Sete tinham doença de Crohn, } 7 \\
\text { apresentavam colite ulcerativa e um não } \\
\text { tinha IBD classificável. A terapia biológica } \\
\text { foi indicada devido à doença refratária } \\
\text { convencional em todos os pacientes. Todos } \\
\text { os pacientes receberam tratamento } \\
\text { combinado com medicamentos } \\
\text { imunossupressores. Uma resposta clínica } \\
\text { favorável foi observada em } 93 \% \text { após a } \\
\text { terapia de indução e } 73 \% \text { apresentaram } \\
\text { remissão endoscópica/imaginológica após } \\
3-6 \text { meses. Apenas um paciente apresentou } \\
\text { efeitos colaterais associados à terapia } \\
\text { biológica, o que não resultou na } \\
\text { descontinuação ou interrupção do do } \\
\text { tratamento. }\end{array}$ \\
\hline
\end{tabular}

\section{DISCUSSÃO}

A partir da leitura atenta dos artigos, bem como da análise descritiva dos resultados encontrados nos mesmos, a discussão pautou-se na terapia biológica na doença de Crohn e enfoque positivo nos resultados farmacoterapêuticos.

A citocina TNF- $\alpha$ atua no recrutamento e ativação de células do sistema imunológico, assim como na ativação de fibroblastos e células endoteliais, eventos que respaldam sua importância no agravamento do processo inflamatório de mucosas, a exemplo da intestinal, no caso da DC (TORRES et al., 2009).

$\mathrm{Na}$ condução dos eventos relacionados à $\mathrm{TB}$, dois estudos foram importantes para respaldar fortemente o uso do IFX como potente agente farmacológico no tratamento da DC. O primeiro deles foi o estudo randomizado e multicêntrico $\mathrm{REACH}$, que visava avaliar a segurança e a eficácia desta alternativa terapêutica em 
pacientes com faixa etária pediátrica, apresentando tal condição patológica em estágios compreendidos como moderado e severo. O segundo, por sua vez, de delineamentos randomizado e cego, conhecido como SONIC, demonstrava que o referido fármaco, em monoterapia ou combinado com azatioprina (AZA), exibia, em pacientes com DC moderada a grave, uma taxa significativamente mais elevada de remissão clínica sem corticosteroides do que àquela observada com a $A Z A$ isolada (MARQUES et al., 2012).

Em suma e via de regra, segundo Rojas T. et al. (2015), o tratamento para a DC se pauta nos seguintes objetivos: alcance da resposta remissiva clínica sem a utilização de corticosteroides, cicatrização da mucosa intestinal, manutenção da homeostase gastrintestinal e prevenção de procedimentos cirúrgicos e hospitalizações.

Levando em consideração o exposto por Malheiros et al. (2009), a TB ganhou espaço quando a terapêutica convencional se mostrou ineficaz para a diminuição dos surtos de exacerbação e suas frequências na DC. Assim, embora existam várias classes farmacológicas utilizadas para esta finalidade, como os salicilatos e seus derivados, corticosteroides, antibióticos e imunossupressores, os clínicos por vezes se deparam com uma questão desapontadora, a qual se configura como a não responsividade ao tratamento com tais entidades terapêuticas.

Em um estudo realizado por Yamamoto; Chiba; Matsumoto (2015), foi demonstrado que a TB tem efeito positivo na redução do estresse oxidativo, uma vez que a DC, por desencadear a infiltração de neutrófilos na parede intestinal, promove o dano oxidativo via liberação de espécies reativas de oxigênio (EROs), associadas com peroxidação lipídica, oxidação proteica e inativação enzimática.

Já em estudo feito por Marques et al. (2012), evidenciou-se que todos os cinco doentes pediátricos com DC, que ingressaram em protocolo terapêutico com IFX por via endovenosa, no Hospital Garcia de Orta, apresentavam a doença em sua forma ativa e refratária à terapêutica convencional. Após inserção de agente anti-TNF- $\alpha$, seja por substituição aos medicamentos utilizados anteriormente ou em terapia combinada a esses ou outros, no esquema de tratamento que fora avaliado, tornou-se nítida a melhora do quadro clínico dos indivíduos assistidos, uma vez que, 
além de demonstrar boa tolerabilidade, tal fármaco mostrou eficácia no controle da doença, com a indução da remissão.

Gellona V et al. (2006), investigando a eficácia do IFX no tratamento da DC, num grupo de 12 pacientes (10 mulheres e 2 homens, com faixa etária variando entre 16 a 63 anos), verificaram que a primeira dose administrada do fármaco induziu uma resposta clínica favorável em $75 \%$ dos tratados, enquanto foi obtida uma taxa de remissão equivalente a $58 \%$ dos mesmos. Em adição a isso, observouse também que apenas 2 pacientes apresentaram efeitos adversos, porém nenhum foi de natureza grave ou secundário à administração.

Torres et al. (2009), ao enfatizarem o papel do IFX no tratamento da DC, afirmam que ele não atua apenas através do bloqueio direto do TNF- $\alpha$ solúvel, mas também na indução do processo apoptótico de linfócitos $T$, recuperação da integridade da barreira epitelial e indução da mobilidade de fibroblastos presentes no intestino, o que culmina na cicatrização das lesões.

Steinwurz (2003) analisando 44 pacientes tratados com o fármaco, na dose de $5 \mathrm{mg} / \mathrm{kg}$, via infusão endovenosa, observou que 30 deles obtiveram melhora $(68,2 \%)$, com diminuição bastante considerável nos níveis de atividade da doença, e $8(51,7 \%)$, dos 14 que eram portadores de fístulas decorrentes de complicações da DC, também apresentaram bons resultados, já que houve o fechamento ou diminuição drástica do processo de drenagem das mesmas. Assim, fica claro que o IFX é uma alternativa terapêutica viável para indivíduos não responsivos a outras abordagens farmacológicas, como os corticosteroides, ou que apresentem complicações fistulares associadas.

Para Kotze et al. (2014b), outro fator que merece atenção no trajeto a ser escolhido, na prática clínica, é o grau de complexidade da fístula (simples ou complexa). Se simples, como as fístulas interesfincterianas superficiais, fistulotomias associadas à terapia medicamentosa convencional (antibióticos e imunomoduladores) já demonstram desfechos favoráveis. No entanto, quando complexas, faz-se necessária a combinação de colocação do seton e posterior inserção da TB, uma vez que este foi o caminho apontado como exitoso, no presente estudo, para a remissão perianal completa, corroborando os achados literários já publicados para tal problemática. 
Luna-Chadid et al. (2004) também evidenciam o papel do IFX na promoção de respostas clínicas favoráveis ou remissivas em pacientes com DC fistulizante, atentando para os efeitos adversos ao tratamento, os quais, quando observados sob uma ótica quanti-qualitativa, são infrequentes, leves e responsivos à terapia apropriada, sem a ocorrência de mortes.

Devido às reações imunogênicas que alguns pacientes apresentam ao IFX, muitas vezes associadas à composição molecular do mesmo, que contém uma porção murina, o fármaco de escolha para este tipo de situação é o ADA (KOTZE et al., 2011).

Além de corroborarem que o ADA é eficaz e seguro, López Palacios et al. (2008) ainda reforçam o seu uso como uma potente alternativa terapêutica em casos onde se tenha observado perda da resposta ou intolerância ao IFX.

De acordo com Barreiro-De-Acosta; Lorenzo; Domínguez-Muñoz (2012), a TB demonstra resultados promissores no tratamento da maioria das manifestações extra-intestinais (MEls), mesmo essas tendo evolução não paralela à DC. Tais autores apontam ainda que, dois de cada três pacientes com MEls, relacionadas à $D C$, se beneficiam com o tratamento com ADA, o que reafirma que seu papel farmacológico não se destina apenas na terapia de pacientes virgens de IFX ou que não respondem a esse. Para corroborar isso, foram alcançados como resultados, no presente estudo, remissão ou resposta parcial de $61,3 \%$ e $71,4 \%$, respectivamente, em portadores de artrite periférica e espondilite anquilosante, decorrentes de complicações da condição patológica em questão.

O estudo desenvolvido por Kotze et al. (2014a) demonstrou que a eficácia de ADA, em induzir remissão clínica, não se tornou tão prejudicada, a ponto de tornarse nula ou próxima a isso, em pacientes que utilizaram previamente IFX, embora tenha sido maior naqueles que eram considerados virgens de IFX (69,23\% nos que receberam tratamento prévio com IFXversus (vs) 94,59\% nos virgens de IFX). Ademais, tais autores também trazem que fármacos corticosteroides, embora possam induzir a remissão, não são tão efetivos clinicamente, isso se observados alguns aspectos, como sua toxicidade e baixa capacidade de manter o estado remissivo. 
Em estudo realizado por López Palacios et al. (2008), onde se analisou 22 pacientes tratados com ADA, dos quais 16 apresentavam DC luminal e 6 exibiam DC fistulizante perianal ativa, pôde-se comprovar a eficácia de tal fármaco em promover remissão completa ou resposta parcial após indução. Dos indivíduos sob análise, considerando tais respostas, respectivamente, $25 \%$ estavam configurados no primeiro tipo (remissão completa) e $56,3 \%$ no segundo (resposta parcial), com percentuais de pacientes, no que diz respeito à manutenção da resposta clínica, variando no período de $12(71,6 \%), 18(53,7 \%)$ e 48 meses $(35,8 \%)$.

Kotze et al. (2011) relatam que, dos 54 pacientes analisados em seu estudo, 29 apresentavam dependência a corticosteroides, o que pode trazer impactos negativos na eficácia da TB, uma vez que houve uma mudança tardia no esquema terapêutico. Esta situação geralmente associa-se a casos de pior prognóstico no futuro. Adicionalmente, verificou-se também que, embora os estudos de eficácia associada só tenham maior segurança com relação ao IFX (IFX + AZA), foi visto ainda que ADA em associação com AZA apresenta resultados positivos, pois, desses mesmos 54 indivíduos, 48 foram tratados dessa maneira.

Rojas T. et al. (2015) também sustentam a base de que a TB, em associação com AZA, apresenta resultados farmacoterapêuticos, além de clínicos, maiores, já que, via observação em estudo desenvolvido por tais, obteve-se uma taxa de melhora de $73 \%$ em aspectos concernentes à resposta endoscópica. Aliado a esse benefício, houve remissão clínica sustentada e redução de hospitalizações, o que vai de encontro aos objetivos do tratamento da DC.

\section{CONCLUSÃO}

A DC, além de promover mudanças potencialmente negativas na qualidade de vida dos seus portadores, ainda se constitui como um desafio para o estabelecimento preciso de suas bases fisiopatológicas, diagnósticas e terapêuticas, já que, por não ter uma patogênese totalmente esclarecida, várias são as questões que permeiam os aspectos investigativos. 
É satisfatório, no entanto, observar o quanto tal modalidade terapêutica, através dos fármacos IFX e ADA, tem promovido resultados terapêuticos positivos na vida dos pacientes com DC, o que se reflete em melhores aspectos de qualidade de vida e indução de remissão clínica e sua manutenção. Nesta perspectiva, investigar o assunto, bem como divulgá-lo mediante a tudo o que foi obtido, é de grande relevância.

Em suma, o profissional farmacêutico, assim como toda a equipe multidisciplinar de saúde, cumprindo as atribuições que the são conferidas e respeitando as demais, podem contribuir com a obtenção de melhores desfechos clínicos. Para tal profissional, através dos serviços de farmácia clínica e atenção farmacêutica, esse objetivo pode ser alcançado, por exemplo, com medidas de monitoramento de parâmetros bioquímicos, ajuste de esquemas terapêuticos, observação e notificação de efeitos colaterais e reações adversas, entre outras.

\section{REFERÊNCIAS BIBLIOGRÁFICAS}

BACK, Ivi Ribeiro et al. BODY COMPOSITION IN PATIENTS WITH CROHN'S DISEASE AND ULCERATIVE COLITIS. Arq. Gastroenterol. São Paulo, v. 54, n. 2, p. 109-114, Jun. 2017. Disponível em: <http://www.scielo.br/scielo.php?script=sci arttext\&pid=S000428032017000200109\&lng=en\&nrm=iso>. Acesso em: 02 Jul. 2018. http://dx.doi.org/10.1590/s0004-2803.201700000-02.

BARREIRO-DE-ACOSTA, Manuel; LORENZO, Aurelio; DOMÍNGUEZ-MUÑOZ, J. Enrique. Efficacyofadalimumab for thetreatmentofextraintestinalmanifestationsofCrohn'sdisease. Rev. esp. enferm. dig., Madrid, v. 104, n. 9, p. 468-472, Set. 2012. Disponível em: $<$ http://scielo.isciii.es/scielo.php?script=sci_arttext\&pid=S1130-
$01082012000900004 \& \operatorname{lng}=$ es\&nrm=iso $>$. Acesso
em:
20
Jul.
2018.

http://dx.doi.org/10.4321/S1130-01082012000900004.

BECHARA, Cristiane de Souza et al. Montreal classificationofpatientoperated for Crohn'sdiseaseandidentificationofsurgicalrecurrencepredictors. Rev. Col. Bras. Cir. Rio de Janeiro, v. 42, n. 2, p. 97-104, Abr. 2015. Disponível em: $<$ http://www.scielo.br/scielo.php?script=sci_arttext\&pid=S010069912015000300097\&lng=en\&nrm=iso>. Acesso em: 17 Jul. 2018. http://dx.doi.org/10.1590/0100-69912015002006.

DENADAI, Rafael; TEIXEIRA, Fábio Vieira; SAAD-HOSSNE, Rogério. The onsetofpsoriasisduringthetreatmentofinflammatoryboweldiseaseswithinfliximab:

shouldbiologicaltherapybesuspended?. Arq. Gastroenterol. São Paulo, v. 49, n. 2, p. 172176, Jun. 2012. Disponível em: <http://www.scielo.br/scielo.php?script=sci_arttext\&pid=S000428032012000200014\&lng=en\&nrm=iso>. Acesso em: $03-$ Jun. 2018. http://dx.doi.org/10.1590/S0004-28032012000200014.

GELLONA V, José et al. Infliximabeneltratamiento de laenfermedad de Crohn: Estudio 
preliminar. Rev. méd. Chile, Santiago, v. 134, n. 3, p. 320-325, Mar. 2006. Disponível em: $<$ https://scielo.conicyt.cl/scielo.php?script=sci_arttext\&pid=S0034-

98872006000300008\&lng=es\&nrm=iso>. Acesso em: 20 Jul. 2018. http://dx.doi.org/10.4067/S0034-98872006000300008.

HARDT, Marcelo Rassweiler et al. Epidemiological profile of 175 patientswithCrohn'sdiseasesubmittedtobiologicaltherapy. J. Coloproctol. (Rio J.), Rio de Janeiro, v. 32, n. 4, p. 395-401, Dez. 2012. Disponível em: $<$ http://www.scielo.br/scielo.php?script=sci_arttext\&pid=S2237-

93632012000400006\&lng=en\&nrm=iso>. $\quad$ Acesso em: 2018. http://dx.doi.org/10.1590/S2237-93632012000400006.

KOTZE, Paulo Gustavo et al. ADALIMUMAB FOR MAINTENANCE THERAPY FOR ONE YEAR IN CROHN'S DISEASE: resultsof a Latin American single-center observationalstudy. Arq. Gastroenterol., São Paulo, v. 51, n. 1, p. 39-45, Mar. 2014a. Disponível em: $<$ http://www.scielo.br/scielo.php?script=sci_arttext\&pid=S0004-

28032014000100039\&Ing=en\&nrm=iso $>$. Acesso em: 20 Jul. 2018. http://dx.doi.org/10.1590/S0004-28032014000100009.

KOTZE, Paulo Gustavo et al. Adalimumab in theinductionofCrohn'sdiseaseremission: resultsof a Brazilianmulticenter case series. J. Coloproctol. (Rio J.), Rio de Janeiro, v. 31, n. 3, p. 233240, Set. 2011. Disponível em: <http://www.scielo.br/scielo.php?script=sci_arttext\&pid=S223793632011000300002\&lng=en\&nrm=iso>. Acesso em: 19 Jul. 2018. http://dx.doi.org/10.1590/S2237-93632011000300002.

KOTZE, Paulo Gustavo et al. PERIANAL COMPLETE REMISSION WITH COMBINED THERAPY (SETON PLACEMENT AND ANTI-TNF AGENTS) IN Crohn's DISEASE: a Brazilianmulticenterobservationalstudy. Arq. Gastroenterol. São Paulo, v. 51, n. 4, p. 284-289, Dez. 2014b. Disponível em: <http://www.scielo.br/scielo.php?script=sci_arttext\&pid=S000428032014000400284\&lng=en\&nrm=iso>. Acesso em: 19 Jul. 2018. http://dx.doi.org/10.1590/S0004-28032014000400004.

LARANJEIRA, Nuno et al. ORAL MUCOSA LESIONS AND ORAL SYMPTOMS IN INFLAMMATORY BOWEL DISEASE PATIENTS. Arq. Gastroenterol. São Paulo, v. 52, n. 2, p. 105-110, Jun. $2015 . \quad$ Disponível em: $<$ http://www.scielo.br/scielo.php?script=sci_arttext\&pid=S0004-

28032015000200105\&lng=en\&nrm=iso>. Acesso em: 15 Jul. 2018. http://dx.doi.org/10.1590/S0004-28032015000200006.

LAW, Siu-tong; LI, Kin Kong. GENDER-RELATED DIFFERENCES IN CLINICAL COURSE OF CROHN'S DISEASE IN AN ASIAN POPULATION: a retrospectivecohort review. Arq. Gastroenterol. São Paulo, v. 51, n. 2, p. 90-96, Jun. 2014. Disponível em: $<$ http://www.scielo.br/scielo.php?script=sci_arttext\&pid=S0004-

28032014000200090\&Ing=en\&nrm=iso>. Acesso em: 16 Jul. 2018. http://dx.doi.org/10.1590/S0004-28032014000200004.

LÓPEZ PALACIOS, N. et al. Tratamiento de inducción y mantenimientoconadalimumabenlaenfermedad de Crohn: unestudioabierto. Rev. esp. enferm. dig. Madrid, v. 100, n. 11, p. 676-681, Nov. 2008. Disponível em: $<$ http://scielo.isciii.es/scielo.php?script=sci_arttext\&pid=S1130-

01082008001100002\&lng=es\&nrm=iso>. Acesso em: 23Ago. 2018.

LUNA-CHADID, $M$. et al. Predictorsof response toinfliximab in patientswithfistulizingCrohn'sdisease. Rev. esp. enferm. dig., Madrid, v. 96, n. 6, p. 379384, Jun. 2004. Disponível em: <http://scielo.isciii.es/scielo.php?script=sci_arttext\&pid=S1130$01082004000600003 \&$ Ing=es\&nrm=iso>. Acesso em: 02 Set. 2018.

MALHEIROS, Anna Paula Rocha et al. Tratamento da doença de Crohn com infliximabe: 
primeira opção?. ABCD, arq. bras. cir. dig. São Paulo, v. 22, n. 2, p. 101-104, Jun. 2009. Disponivel em: <http://www.scielo.br/scielo.php?script=sci_arttext\&pid=S010267202009000200007\&Ing=en\&nrm=iso>. Acesso em: 19 Jul. 2018. http://dx.doi.org/10.1590/S0102-67202009000200007.

MARQUES, Rita et al. Uso do infliximab na consulta de gastrenterologia pediátrica. J PortGastrenterol. Lisboa, v. 19, n. 3, p. 126-129, Maio 2012. Disponível em $<$ http://www.scielo.mec.pt/scielo.php?script=sci_arttext\&pid=S0872-

81782012000300004\&lng=pt\&nrm=iso>. Acesso em: 15 Ago. 2018.

NOGUEIRA, Islaine Martins et al. ASSESSMENT OF THE RESPONSE OF PATIENTS WITH CROHN'S DISEASE TO BIOLOGICAL THERAPY USING NEW NON-INVASIVE MARKERS: lactoferrinandcalprotectin. Arq. Gastroenterol. São Paulo, v. 50, n. 2, p. 130-137, Jun. 2013. Disponivel em: <http://www.scielo.br/scielo.php?script=sci_arttext\&pid=S000428032013000200130\&lng=en\&nrm=iso $>$. Acesso em: 16 Set. 2018. http://dx.doi.org/10.1590/S0004-28032013000200022.

SALES-CAMPOS, $\mathrm{H}$. et al. Classicalandrecentadvances in thetreatmentofinflammatoryboweldiseases. Braz J MedBiol Res, Ribeirão Preto, v. 48, n. 2, p. 96-107, Fev. 2015. Disponível em: $<$ http://www.scielo.br/scielo.php?script=sci_arttext\&pid=S0100-

879X2015000200096\&lng=en\&nrm=iso>. Acesso em: 18 Jul. 2018. http://dx.doi.org/10.1590/1414-431X20143774.

SANTOS JÚNIOR, Júlio César Monteiro. Crohn'sDisease: currentstateofbiologicaltherapy. J. Coloproctol. (Rio J.), Rio de Janeiro, v. 31, n. 4, p. 407-418, Dez. 2011. Disponível em: $<$ http://www.scielo.br/scielo.php?script=sci_arttext\&pid=S2237-

93632011000400017\&lng=en\&nrm=iso>. Acesso em: 02 Ago. 2018. http://dx.doi.org/10.1590/S2237-93632011000400017.

SCOLARO, Bruno Lorenzo et al. T300A GENETIC POLYMORPHISM: a susceptibilityfactor for Crohn'sdisease?. Arq. Gastroenterol. São Paulo, v. 51, n. 2, p. 97-101, Jun. 2014. Disponível em: $\quad<h t t p: / / w w w . s c i e l o . b r / s c i e l o . p h p ? s c r i p t=s c i \_a r t t e x t \& p i d=S 0004-$ 28032014000200097\&lng=en\&nrm=iso>. Acesso em: 19 Jun. 2018. http://dx.doi.org/10.1590/S0004-28032014000200005.

STEINWURZ, Flavio. Experiência clínica com o uso de infliximab em 44 portadores de doença de Crohn. Arq. Gastroenterol. São Paulo, v. 40, n. 3, p. 198-200, Set. 2003. Disponível em: $<$ http://www.scielo.br/scielo.php?script=sci_arttext\&pid=S0004-

$28032003000300012 \& \mathrm{lng}=\mathrm{en \& nrm=iso>}$. Acesso em: 19 Jul. 2018. http://dx.doi.org/10.1590/S0004-28032003000300012.

T., Catalina Rojas et al. Experiencia del uso de terapia biológica en pacientes conenfermedadinflamatoria intestinal enun hospital público. Gastroenterol. Latinoam, Santiago, p.94-100, 21 Abr. 2015.2 Disponível em: <http://gastrolat.org/DOI/PDF/10.0716/gastrolat2015n200003.pdf>. Acesso em: 19 Jul. 2018.

TOMAZONI, Esmirrá Isabella; BENVEGNÚ, Dalila Moter. SYMPTOMS OF ANXIETY AND DEPRESSION, AND QUALITY OF LIFE OF PATIENTS WITH CROHN'S DISEASE. Arq. Gastroenterol. São Paulo, v. 55, n. 2, p. 148-153, Jun. 2018. Disponível em: $<$ http://www.scielo.br/scielo.php?script=sci_arttext\&pid=S0004-

28032018000200148\&Ing=en\&nrm=iso>. Acesso em: $17 \quad$ Jul. 2018. http://dx.doi.org/10.1590/s0004-2803.201800000-26.

TORRES, Ulysses dos Santos et al. Infliximabe na doença de crohn: experiência clínica de um centro terciário paulista. Rev bras. colo-proctol. Rio de Janeiro, v. 29 , n. 1 , p. $38-45$, Mar. 2009. Disponivel em: <http://www.scielo.br/scielo.php?script=sci_arttext\&pid=S010198802009000100005\&lng=en\&nrm=iso>. Acesso em: $19 \quad$ Jul. 2018. 
http://dx.doi.org/10.1590/S0101-98802009000100005.

VICTORIA, Carlos Roberto; SASSAK, Ligia Yukie; NUNES, Hélio Rubens de Carvalho. Incidenceandprevalence rates ofinflammatoryboweldiseases, in midwesternof São Paulo State, Brazil. Arq. Gastroenterol. São Paulo, v. 46, n. 1, p. 20-25, Mar. 2009. Disponível em: $<$ http://www.scielo.br/scielo.php?script=sci_arttext\&pid=S0004-

28032009000100009\&lng=en\&nrm=iso>. . Acesso em: 02 Jul. 2018. http://dx.doi.org/10.1590/S0004-28032009000100009.

YAMAMOTO, Kazunari; CHIBA, Toshimi; MATSUMOTO, Takayuki. Effectof tumor necrosisfactor- $\alpha$ antagonistsonoxidative stress in patientswithCrohn'sdisease. World JournalOfGastroenterology, [s.I.], v. 21, n. 35, p.10208-10214, 21 Set. 2015. BaishidengPublishingGrouplnc. Disponível em: <https://www.ncbi.nlm.nih.gov/pmc/articles/PMC4572802/>. Acesso em: 19 Jul. 2018. http://dx.doi.org/10.3748/wjg.v21.i35.10208. 